\title{
Reversible Retinopathy Associated with Fibroblast Growth Factor Receptor Inhibitor
}

\author{
Saagar N. Patel Mona L. Camacci Esther M. Bowie \\ Department of Ophthalmology, Penn State College of Medicine, Hershey, PA, USA
}

\section{Keywords}

Fibroblast growth factor receptor - Fibroblast growth factor receptor retinopathy - Optical coherence tomography · Mitogen-activated protein kinase $\cdot$ Mitogen-activated protein kinase kinase associated retinopathy

\begin{abstract}
We present a case of reversible, pseudovitelliform lesions while a patient was taking pembrolizumab (PDL-1 inhibitor) and erdafitinib (pan-fibroblast growth factor receptor inhibitor) outside of clinical trial protocols. A 61-year-old patient with 3 days of metamorphopsia was found to have pseudovitelliform lesions in both eyes 6 weeks after initiation of erdafitinib. After discontinuation of this drug, his visual complaints resolved and his lesions decreased on optical coherence tomography. To our knowledge, this is the first case depicting reversible macular lesions with use of this newly approved medication outside of clinical trial protocols.
\end{abstract}

\section{Introduction}

Fibroblast growth factor receptors (FGFRs) play an essential role in key eukaryotic biological signaling networks that closely regulate cell proliferation, survival, migration, and differentiation [1]. Thus, FGFR inhibitors are novel targeted chemotherapies in development for many cancers. Their downstream effects inhibit the mitogen-activated protein kinase (MAPK) and phosphatidylinositol-3-kinase pathway. In the eye, FGFR signaling is vital to the development, survival, stress response, and repair of retinal pigment epithelium (RPE) cells; ganglion cell maturation and survival; and regulation of Müller cells in the retina [2, 3]. 
MAPK inhibition with mitogen-activated protein kinase kinase (MEK) inhibitors leads to a characteristic, reversible MEK-associated retinopathy (MEKAR) [4-7]. We present a patient being treated with an FGFR inhibitor for metastatic urothelial cancer with reversible, bilateral pseudovitelliform lesions similar to MEKAR outside of clinical trial protocols.

\section{Case Report}

A 61-year-old male with metastatic bladder cancer (fibroblast growth factor receptor 2 F276C mutation) presented to clinic with 3 days of metamorphopsia in both eyes 6 weeks after initiation of erdafitinib (pan-fibroblast growth factor inhibitor; FGFR inhibitor) $8 \mathrm{mg}$ daily. The patient's oncological regimen included erdafitinib $6 \mathrm{mg}$ daily and pembrolizumab (programmed death ligand-1 inhibitor; PD-L1 inhibitor) $200 \mathrm{mg}$ weekly. He denied use of concurrent steroid medications. He was referred by his oncologist for concerns of ocular side effects from his cancer therapy regimen. The patient was instructed by his oncologist to reduce his erdafitinib dosage from $8 \mathrm{mg}$ to $6 \mathrm{mg}$ daily with onset of ocular symptoms.

Best corrected visual acuity was 20/25 in the right eye and 20/30 in the left eye. Intraocular pressures were within normal limits; the anterior segment exam was significant for mild nuclear sclerosis cataracts in both eyes. On dilated fundus examination, bilateral yellow foveal vitelliform elevations without other fluid foci were noted; there was no vitreous inflammation noted. Fundus near-infrared reflectance demonstrated bilateral solitary central hyporeflective lesions with a rim of hyper-reflectivity (Fig. 1). Optical coherence tomography (OCT) confirmed a small neurosensory retinal detachment and hyper-reflective subretinal material in each eye without choroidal thickening (Fig. 2). After ocular findings were discussed with the patient's oncologist, the decision was made to discontinue erdafitinib by oncology; weekly pembrolizumab was continued. Within several days of discontinuing the FGFR inhibitor, the patient reported resolution of his metamorphopsia. On follow-up 22 days later, best corrected visual acuity was 20/25 in the right eye and 20/25 in the left eye. Repeat nearinfrared reflectance demonstrated reduction of bilateral central lesions with hyper-reflective foci in the previously central areas of hypo-reflectance (Fig. 3). OCT imaging confirmed resolution of small neurosensory retinal detachments and decrease in subretinal hyper-reflective material in both eyes (Fig. 4).

\section{Discussion}

We report a case of reversible, bilateral symptomatic pseudovitelliform lesions with oral erdafitinib outside of a clinical trial protocol. Erdafitinib and pembrolizumab were administered by his oncologist under an experimental treatment protocol.

Erdafinitib is an oral FGFR 1-4 tyrosine kinase inhibitor that allows for targeted chemotherapy aimed at decreasing tumor cell survival, replication, migration, and differentiation [1]. It was granted accelerated approval by the US Food and Drug Administration for treatment of advanced urothelial bladder carcinomas with FGFR2 or FGFR3 mutations on April 12, 2019.

Prensky et al. [8] were the first to demonstrate reversible macular lesions on OCT resembling MEKAR during phase II clinical studies of erdafitinib in 2 patients who presented with blurred vision. One patient experienced subfoveal retinal detachments with hyperreflective subretinal material 2-3 weeks after the patient's dose was escalated from $8 \mathrm{mg}$ to $9 \mathrm{mg}$ of erdafitinib. The subfoveal retinal detachments and hyper-reflective subretinal material resolved at the 1 month follow-up after discontinuing erdafitinib [8]. The patient

\section{Karger'}




\section{Case Reports in Ophthalmology}

Fig. 1. Fundus near-infrared reflectance of the right (a) and left (b) eye demonstrating a central hypo-reflective lesion with rim of hyper-reflectivity.
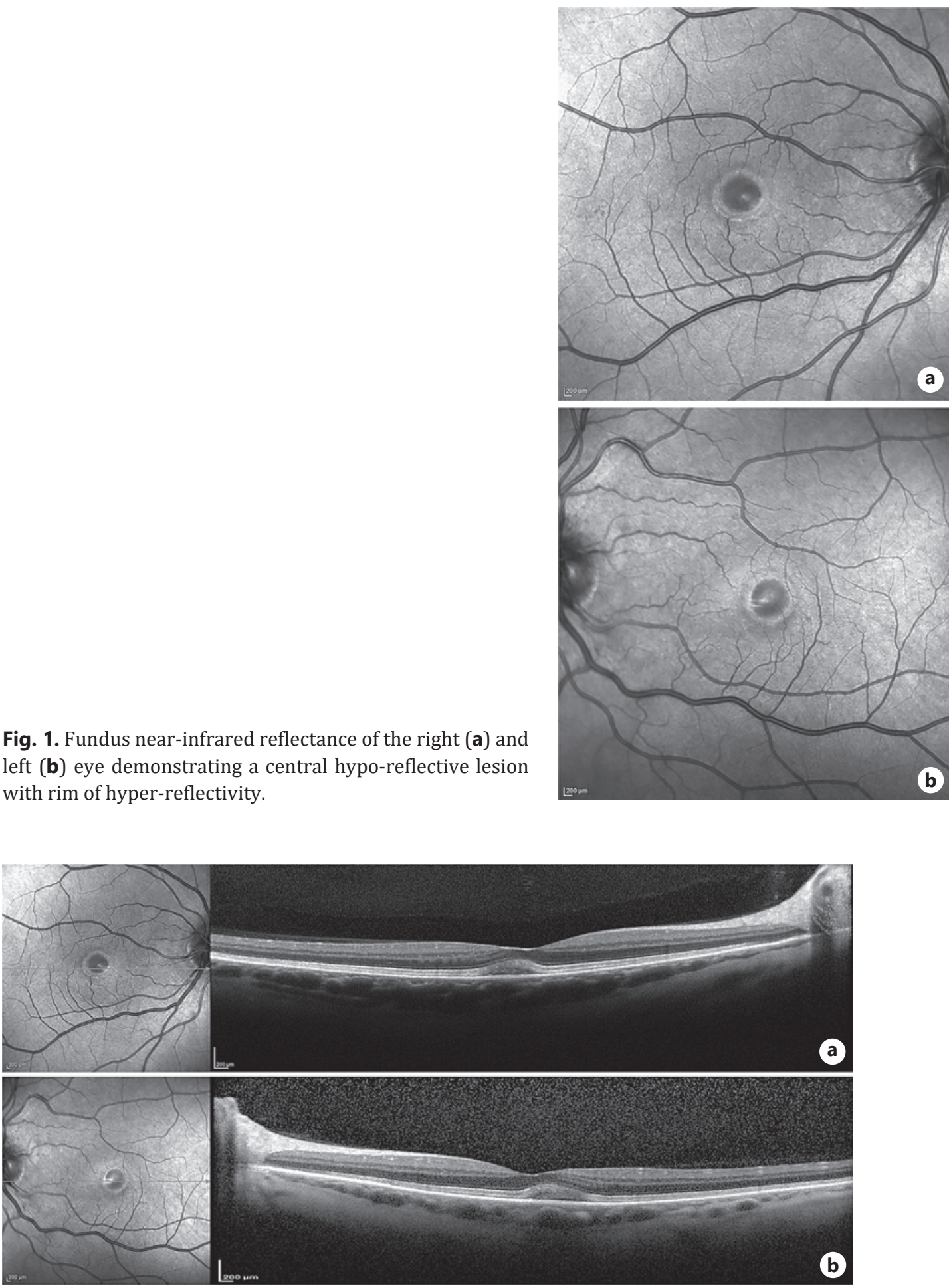

Fig. 2. Optical coherence tomography images of right (a) and left (b) eye demonstrating hyper-reflective, subretinal material with trace subretinal fluid and without choroidal thickening ( $229 \mu \mathrm{m}$ in both eyes).

was started on a lower dose regimen and did not have any additional symptoms or findings on follow-up [8]. The second patient developed bilateral, multiple foci of retinal detachments with thickening of the photoreceptor layer 3 weeks after dose escalation to erdafitinib 


\section{Case Reports in Ophthalmology}

Fig. 3. Fundus near-infrared reflectance of the right (a) and left (b) eye demonstrating foci of hyper-reflectivity in area of previously central hypo-reflective lesion.
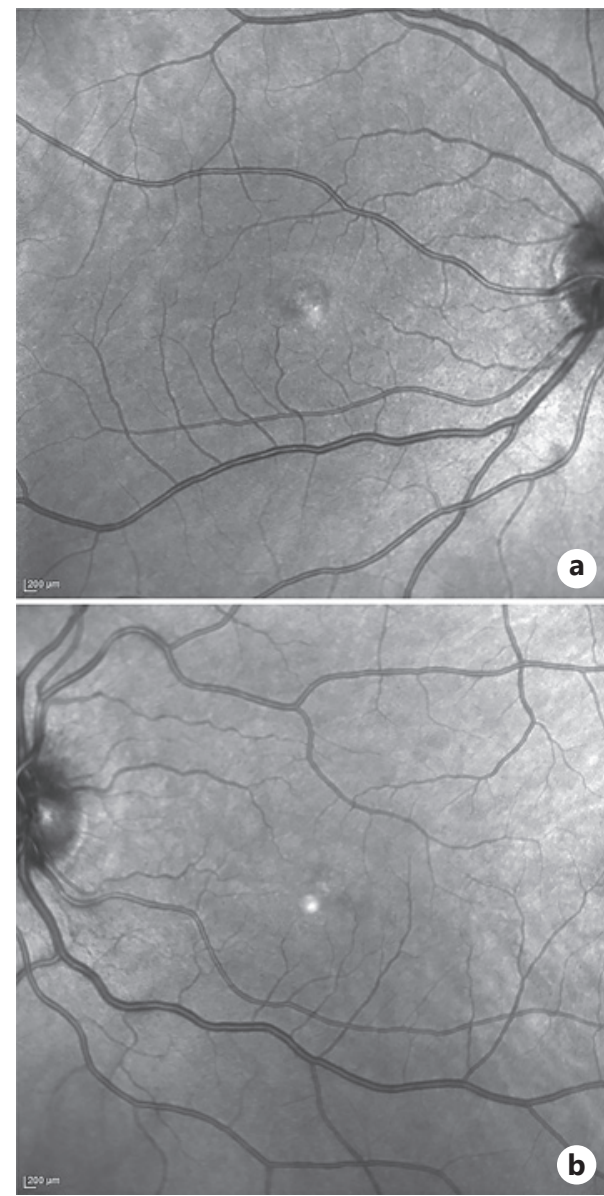

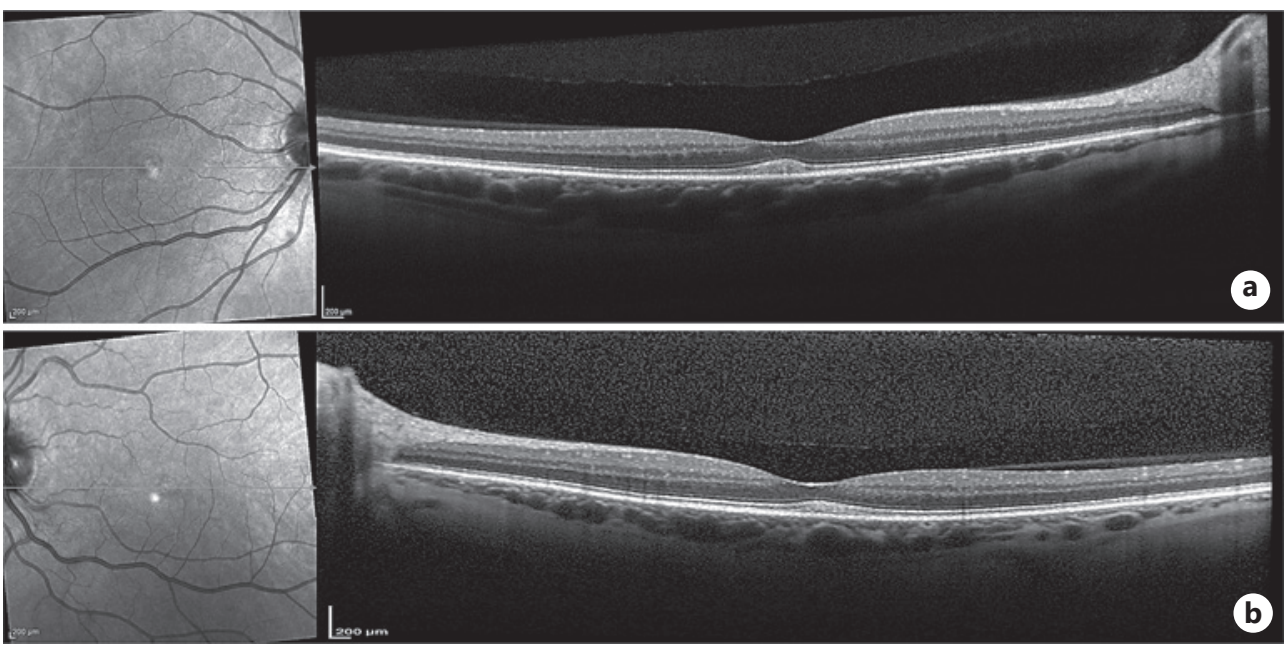

Fig. 4. Optical coherence tomography images of right (a) and left (b) eye demonstrating decrease of previous hyper-reflective, subretinal material, and resolution of subretinal fluid. Choroidal thickness remained constant at $229 \mu \mathrm{m}$ in both eyes.

$8 \mathrm{mg}$ daily; erdafitinib was discontinued and the lesions decreased at the 2 week followup. The patient had recurrence of the lesions when restarted at a lower dose of $6 \mathrm{mg}$ daily [8]. 
Parikh et al. demonstrated a case of FGFR-associated retinopathy in which a patient developed thickened subfoveal ellipsoid (EZ) and interdigitation zones which worsened to subfoveal retinal detachments 3 months following initiation of erdafitinib for poorly differentiated gastric adenocarcinoma [9]. Although the patient developed shallow subfoveal retinal detachments, the patient remained asymptomatic throughout the entire course of erdafitinib use with visual acuity of $20 / 20$ OU at 3 months so erdafitinib was continued with plans for close monitoring of the patient [9].

The OCT findings in our patient resemble those depicted by the first case by Prensky et al. [8] and the case presented by Parikh et al. [9]. Our patient's subretinal hyper-reflective material decreased over a 1 month period after drug cessation. Erdafinitib was discontinued in our patient due to lack of efficacy and the patient's poor tolerance of extraocular side effects. Remarkably, the patient reported by Parikh et al. [9] remained visually asymptomatic throughout the duration of erdafitinib use, suggesting that FGFR-associated retinopathy may be present in asymptomatic patients who would not otherwise pursue ophthalmologic examination [8]. A phase II trial evaluating another oral pan-FGFR inhibitor, AZD4547, demonstrated signs of MEKAR-like retinopathy in 50\% (12/24) of patients and all were reportedly asymptomatic [10]. Alekseev et al. [11] reported on a patient who developed multifocal serous retinopathy similar to MEKAR while taking another oral pan-FGFR inhibitor, pemigatinib. The patient had a visual acuity of 20/20-2 in the right eye and 20/20-1 in the left eye and reported mild blurring of vision [11]. There are currently no consensus recommendations for FGFR retinopathy screening. Some authors suggest monthly dilated eye examinations for at least the first 4 months of FGFR inhibitor use with further spacing of visits; however, longitudinal OCT analysis of patients on FGFR inhibitors remains to be seen [12].

Longitudinal analysis of foveal and parafoveal OCT images for patients on combined hydroxychloroquine, MEK inhibitor, and BRAF inhibitor showed a transient increase in the EZ to RPE length at approximately 30 days in 9/11 otherwise visually asymptomatic patients, even in those without identifiable MEKAR [13]. Notably, the EZ to RPE length decreased back towards baseline levels at subsequent follow-up 180 days later for those patients [13]. The case presented by Parikh et al. [9] demonstrated continued widening of the EZ-RPE length at the 90 day mark.

Our patient was also taking pembrolizumab immunotherapy for treatment of his cancer. In cases involving the retina, this has been associated with a robust inflammatory response typically manifesting as posterior uveitis and/or optic disc edema [14]. The patient had no signs or symptoms to suggest that he had an increased inflammatory response as reported with vision loss associated with pembrolizumab use.

As FGFR inhibitor use increases, ophthalmologists should be aware of the potential retinal dysfunction these medications may cause. Our case presents a scenario outside of clinical trial protocols in which a patient developed reversible findings similar to MEKAR. As seen in this case, it is important to understand the various presentations of ocular toxicities or dysfunction associated with different medications and to differentiate this from other common ocular pathologies such as central serous chorioretinopathy. This is the third case with ocular imaging showing reversibility of lesions with erdafitinib use. Our patient had mild symptoms and resolving retinopathy on discontinuation of the drug with minimal consequences to his vision. Further cases and mechanistic research may help clarify the phenotype observed with FGFR inhibitors compared to other agents targeting the MAPK pathway.

\section{Acknowledgment}

We thank the patient's family for permission to publish this article and cooperation with data collection. 


\section{Statement of Ethics}

This study protocol was reviewed and the need for approval was waived by the Penn State College of Medicine Institutional Review Board, STUDY00017632. Written informed consent to allow collection, reporting, and publishing of the clinical and retinal imaging findings for this case report was obtained from the patient's next of kin. Efforts to maintain anonymity were upheld during drafting of the manuscript.

\section{Conflict of Interest Statement}

None of the authors have any financial disclosures or conflicts of interest to declare.

\section{Funding Sources}

No funding was received for this study.

\section{Author Contributions}

S.N.P. participated in conceiving and designing the manuscript, drafting the manuscript, final editing of the manuscript, and agreeing to accountability of the work. M.C. participated in designing the manuscript, critical editing of the manuscript, final editing of the manuscript, and agreeing to accountability of the work. E.B. participated in conceiving and designing the manuscript, interpreting figures, critical editing of the manuscript, final editing of the manuscript, and agreeing to accountability of the work.

\section{Data Availability Statement}

All data generated or analyzed during this study are included in this article. Further inquiries can be directed to the corresponding author.

\section{References}

1 Loriot Y, Nechhi A, Park SH, Garcia-Donas J, Huddart R, Burgess E, et al. Erdafitinib in locally advanced or metastatic urothelial carcinoma. N Engl J Med. 2019;381:338-48.

2 van der Noll R, Leijen S, Neuteboom GH, Beijnen JH, Schellens JH. Effect of inhibition of the FGFR-MAPK signaling pathway on the development of ocular toxicities. Cancer Treat Rev. 2013;39(6):664-72.

3 Catalani E, Tomassini S, Dal Monte M, Bosco L, Casini G. Localization patterns of fibroblast growth factor 1 and its receptors FGFR1 and FGFR2 in postnatal mouse retina. Cell Tissue Res. 2009;336:423-38.

4 Duncan KE, Chang LY, Patronas M. MEK inhibitors: a new class of chemotherapeutic agents with ocular toxicity. Eye. 2015;29(8):1003-12.

5 McCannel TA, Chmeilowski B, Finn RS, Goldman J, Ribas A, Wainberg ZA, et al. Bilateral subfoveal neurosensory retinal detachment associated with MEK inhibitor use for metastatic cancer. JAMA Ophthalmol. 2014;132:1005-9.

6 Francis JH, Habib LA, Abramson DH, Yannuzzi LA, Heinemann M, Gounder MM, et al. Clinical and morphological characteristics of MEK inhibitor-associated retinopathy: differences from central serous chorioretinopathy. Ophthalmology. 2017;124(12):1788-98.

7 Mendez-Martinez S, Pilar C, Ruiz-Moreno O, Baron NP, Bueno JL, Gil Ruiz MDR, et al. Ocular adverse events associated with MEK inhibitors. Retina. 2019;39(8):1435-50.

8 Prensky C, Marlow E, Gupta M, Sales C, Kiss S, D’Amico DJ. Reversible macular lesions in the setting of oral panfibroblast growth factor inhibitor for the treatment of bladder cancer. J VitreoRetinal Dis. 2017;2(2):111-4. 
9 Parikh D, Eliott D, Kim LA. Fibroblast growth factor receptor inhibitor-associated retinopathy. JAMA Ophthalmol. 2020;138(10):1101-3.

10 Lam WS, Creaney J, Chen FK, Chin WL, Muruganandan S, Arunchalam S, et al. A phase II trial of single oral FGF inhibitor, AZD4547, as second or third line therapy in malignant pleural mesothelioma. Lung Cancer. 2020; 140:87-92.

11 Alekseev 0, Ojuok E, Cousins S. Multifocal serous retinopathy with pemigatinib therapy for metastatic colon adenocarcinoma. Int J Retin Vitr. 2021;7(1):34.

12 Mahipal A, Tella SH, Kommalapati A, Yu J, Kim R. Prevention and treatment of FGFR inhibitor-associated toxicities. Crit Rev Oncol Hematol. 2020;155:103091.

13 Nti AA, Serrano LW, Sandhu HS, Uyhazi KE, Edelstein ID, Zhou EJ, et al. Frequent subclinical macular changes in combined BRAF/MEK inhibition with high-dose hydroxychloroquine as treatment for advanced metastatic BRAF mutant melanoma. Retina. 2019;39(3):502-13.

14 Aaberg MT, Aaberg TM. Pembrolizumab administration associated with posterior uveitis. Retin Cases Brief Rep. 2017;11(4):348-51. 